\title{
Associação entre perineorrafia e problemas perineais, atividades habituais e necessidades fisiológicas afetadas
}

\section{Association between perineorrhaphy and perineal problems, habitual activities, and physiological needs affected}

\author{
Asociación entre perineorrafia y problemas perineales, actividades habituales \\ y necesidades fisiológicas afectadas
}

\section{Luciano Marques dos Santos ${ }^{1}{ }^{(}$, Larissa Madalena da Silva Santos $^{2}{ }^{(}$, Milena Moreira Brandão $^{3} \mathbb{C}^{-}$,

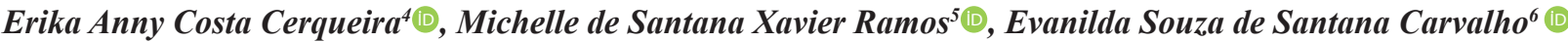

Histórico

Recibido:

23 de febrero de 2018

Aceptado:

19 de abril de 2018

1 Doutorando em Ciências da Saúde pela Universidade Federal de São Paulo, São Paulo, Brasil. Docente do Curso de Graduação em Enfermagem da Universidade Estadual de Feira de Santana, Feira de Santana/BA, Brasil.

2 Graduada em Enfermagem pela Universidade Estadual de Feira de Santana, Feira de Santanal $B A$, Brasil.

3 Acadêmica do $9^{\circ}$ semestre de graduação em enfermagem da Universidade Estadual de Feira de Santana, Feira de Santana/BA, Brasil. Autor de Correspondência: E-mail: milenafsa1995@hotmail.com 4 Académica do $9^{\circ}$ semestre de graduação em enfermagem da Universidade Estadual de Feira de Santana, Feira de Santanal BA, Brasil.

5 Doutoranda em Saúde Coletiva pela Universidade Estadual de Feira de Santana, Feira de Santana/BA, Brasil.

6 Doutora em Enfermagem. Universidade Estadual de Feira de Santana, Feira de Santanal $B A$, Brasil Objetivo: Verificar a associação entre a presença de perineorrafia e problemas perineais, atividades habituais e necessidades fisiológicas afetadas em puérperas no pós-parto vaginal. Materiais e Métodos: Estudo transversal, composto por 870 puérperas com algum tipo de trauma perineal durante o parto vaginal. Os dados foram coletados através da entrevista estruturada e exame físico da região vulvoperineal e submetidos a análise descritiva e inferencial. Resultados: Houve associação estatisticamente significativa entre a episiorrafia e a ocorrência de edema $(\mathrm{p}<0,001)$, ardor $(\mathrm{p}=0,002)$ e dor $(\mathrm{p}=0,002)$. Concernente às atividades habituais e necessidades fisiológicas, mulheres com episiorrafia apresentaram maior dificuldade para vestir-se, deambular, sentar e dormir. Em contrapartida, apresentaram menor dificuldade para urinar e realizar higiene íntima. Discussão: Dentre os problemas perineais, observou-se diferença estatisticamente significativa quanto a ocorrência de edema, ardor e dor entre os grupos, sendo que as mulheres com episiorrafia relataram menos ardor perineal em comparação ao grupo com perineorrafia devido a laceração espontânea. Nesse sentido, preservar a integridade perineal das parturientes é algo necessário e possível na assistência obstétrica, visto que esse tipo de intervenção, por vezes desnecessária, desencadeará desconfortos que irão interferir no seu bem-estar durante o período puerperal. Conclusões: Os dados deste estudo permitiram verificar que há associação entre a perineorrafia e a ocorrência de problemas perineais e alterações para realização de atividades habituais no puerpério imediato.

Introduction: Women in the labor process are exposed to perineal traumas that may be associated with the care. Objective: The work sought to verify the association among the presence of perineorrhaphy and perineal problems, habitual activities and physiological needs affected in puerperae women during vaginal postpartum. Materials and Methods: A cross-sectional study, comprising 870 puerperal women with some type of perineal trauma during vaginal delivery. The data were collected through a structured interview and physical examination of the vulvoperineal region and submitted to descriptive and inferential analyses. Results: A statistically significant association was found between episiorrhaphy and the occurrence of edema $(p<0.001)$, burning $(p=0.002)$, and pain ( $p=$ 0.002 ). Concerning the habitual activities and physiological needs, women with episiorrhaphy had greater difficulty getting dressed, ambulating, sitting, and sleeping. On the other hand, they had less difficulty urinating and performing intimate hygiene. Discussion: Among the perineal problems, a statistically significant difference was noted in the occurrence of edema, burning, and pain between the groups, given that women with episiorrhaphy reported less perineal burning compared to the group with perineorrhaphy due to spontaneous laceration. In this sense, preserving the perineal integrity of parturients is necessary and possible in obstetric care because this type of intervention, sometimes unnecessary, will trigger discomfort that will interfere with their well-being during the puerperal period. Conclusions: The data from this study permitted verifying association between the perineorrhaphy and the occurrence of perineal problems and alterations to carry out habitual activities during immediate puerperium.

Key words: Perineum; Episiotomy; Postpartum Period.

Resumen

Introducción: La mujer en proceso de parto está expuesta a la ocurrencia de traumas perineales que pueden estar asociados a la asistencia. Objetivo: Verificar la asociación entre la presencia de perineorrafia y problemas perineales, actividades habituales y necesidades fisiológicas afectadas en puérperas en el posparto vaginal. Materiales y Métodos: Estudio transversal, compuesto por 870 puérperas con algún tipo de trauma perineal durante el parto vaginal. Los datos fueron recolectados a través de entrevista estructurada y examen físico de la región vulvoperineal y sometidos a análisis descriptivo e inferencial. Resultados: Hubo asociación estadísticamente significativa entre la episiorrafia y la ocurrencia de edema $(p<0,001)$, ardor $(p=0,002)$ y dolor $(p=$ $0,002)$. En cuanto a las actividades habituales y necesidades fisiológicas, las mujeres con episiorrafia presentaron mayor dificultad para vestirse, deambular, sentarse y dormir. En contrapartida, presentaron menor dificultad para orinar y realizarse higiene íntima. Discusión: En los problemas perineales, se observó una diferencia estadísticamente significativa en cuanto a la ocurrencia de edema, ardor y dolor entre los grupos, siendo que las mujeres con episiorrafia refirieron menos ardor perineal en comparación al grupo con perineorrafia debido a la laceración espontánea. En este sentido, preservar la integridad perineal de las parturientas es algo necesario y posible en la asistencia obstétrica, dado que este tipo de intervención, a veces innecesaria, desencadenará en molestias que irán a interferir en su bienestar durante el período puerperal. Conclusiones: Los datos de este estudio permitieron verificar que hay asociación entre la perineorrafia y la ocurrencia de problemas perineales y alteraciones para la realización de actividades habituales en el puerperio inmediato.

Palabras clave: Perineo; Episiotomía; Periodo Posparto.

Como citar este artigo: Santos LM, Santos LMS, Brandão MM, Cerqueira EAC, Ramos MSX, Carvalho ESS. Associação entre perineorrafia e problemas perineais, atividades habituais e necessidades fisiológicas afetadas. Rev Cuid. 2018; 9(2): 2233-44. http://dx.doi.org/10.15649/cuidarte.v9i2.530

(c) (i) (C)2018 Universidad de Santander. Este es un artículo de acceso abierto, distribuido bajo los términos de la licencia Creative Commons Attribution (CC BY-NC 4.0), que permite el uso ilimitado, distribución y reproducción en cualquier medio, siempre que el autor original y la fuente sean debidamente citados. 


\section{INTRODUÇÃO}

A institucionalização do parto tornou o corpo da mulher um objeto de medicalização, visto que houve uma ruptura do cuidado que antes era prestado pelas parteiras num ambiente familiar, onde eram mantidas relações de solidariedade, segurança e confiança ${ }^{1}$. Desta forma, verificase que a medicalização da atenção à mulher em processo parturitivo transformou o parto e o nascimento, considerados eventos de caráter natural, em algo passível da realização de procedimentos e intervenções desnecessárias ${ }^{1-2}$.

Esta realidade é encontrada no Brasil, atual cenário obstétrico que vive uma situação alarmante, no qual a utilização de intervenções desnecessárias é elevada, atribuindo ao parto normal uma conotação dolorosa para as mulheres ${ }^{3}$. Dentre as intervenções utilizadas no parto normal, destacase o uso da episiotomia de rotina como estratégia para a redução das lacerações perineais ${ }^{4}$. Segundo a pesquisa "Nascer no Brasil", a atual taxa deste procedimento nos hospitais brasileiros é de $56 \%{ }^{3}$.

Esse fato não é algo restrito apenas ao cenário nacional, visto que um estudo realizado em 11 países que se encontram em desenvolvimento revelou que entre as intervenções realizadas durante o parto normal, $40 \%$ eram de episiotomias, e estas eram responsáveis pelo aumento da gravidade do dano perineal. Diante disso, podese observar que essa taxa não se distancia do que foi encontrado nos hospitais brasileiros ${ }^{5}$.

A episiotomia é uma incisão cirúrgica realizada na região perineal e na parede posterior da vagina, que comumente é usada para alargar o óstio vaginal e impedir uma laceração espontânea dos músculos perineais ${ }^{6}$.
Outro trauma perineal que pode ocorrer durante o processo parturitivo é a laceração perineal espontânea. As lacerações perineais graves, que se estendem para o complexo do esfíncter anal, embora menos frequentes, são associadas ao aumento do risco de lesão do assoalho pélvico, incontinência fecal e urinária, dor e disfunção sexual com sintomas que podem persistir ou estar presentes muitos anos após o parto 7 .

Dessa forma, observa-se que a mulher em processo parturitivo está exposta a ocorrência de traumas perineais que podem estar associados à assistência, sendo que há destaque para ocorrência de episiotomias.

Uma implicação que o trauma perineal pode trazer para a puérpera é a dor no local da perineorrafia. A dor perineal no pós-parto vaginal é o mais comum dos desconfortos causados pela episiotomia, podendo desencadear alguns sintomas que podem interferir nas necessidades fisiológicas das mulheres bem como no retorno às atividades habituais exercidas. Podem ocorrer, por exemplo, alterações no padrão do sono, apetite, libido, irritabilidade, restrições das atividades funcionais e limitação da mobilidade ${ }^{8}$.

Um estudo realizado com 97 mulheres submetidas a episiotomia revelou que $12,37 \%$ sentiram dor no primeiro dia pós-parto e que $7,21 \%$ continuavam com dor ao exercer suas atividades mesmo após sete dias do parto?.

Problemas perineais como hematomas, fístulas, lesões anais e de mucosa retal ocorrem quando o trauma acontece na região posterior, parede vaginal, fúrcula e musculatura perinea $1^{10}$. Quando ocorrem na região anterior, as lacerações são 
mais superficiais e possuem rápida cicatrização, porém as mulheres queixam-se de desconforto durante a micção. Na região posterior, por sua vez, podem ocorrer implicações de curto prazo, como dor e problemas na cicatrização, e de longo prazo, que estão relacionadas à profundidade da lesão, aos tecidos afetados e à perda sanguínea ${ }^{10}$.

A prevalência de edema e equimose no períneo costuma ser elevada na prática clínica. Um estudo realizado com 54 mulheres que apresentaram laceração de segundo grau ou episiotomia mostrou a ocorrência de $26,2 \%$ e $42,6 \%$ respectivamente. A equimose e o edema apareceram em $44,8 \%$ e $18,5 \%$ dos casos, respectivamente ${ }^{11}$.

Umestudosobrefatoresassociadosàincontinência urinária (IU) feminina apontou a episiotomia como sendo um dos fatores responsáveis pela IU. Este estudo descreveu, ainda, que os eventos obstétricos são um dos principais fatores de risco para o desenvolvimento da IU, correspondendo a cerca de $93,3 \%$ dos $\operatorname{casos}^{12}$.

Outro problema que pode ser causado pelo traumatismo perineal é o distúrbio evacuatório, caracterizado pela perda involuntária das fezes sólidas e líquidas associada ao escape de flatos, tendo a episiotomia como fator de risco para disfunção do diafragma pélvico ${ }^{13}$.

Ao tentar estabelecer o estado da arte sobre o objeto de pesquisa, no período de janeiro a setembro de 2014, na Biblioteca Virtual Em Saúde (BVS), Science Direct e PubMed, utilizandose os descritores "enfermagem obstétrica, parto normal, períneo, episiotomia, período pós- parto e dor", e seus respectivos na língua inglesa e espanhola, foram encontrados 18 artigos. Estes descreviam a ocorrência de dor perineal no pós-parto vaginal associada a episiotomia (14 artigos encontrados), dispareunia (2 artigos), incontinência urinária (1 artigo) e algumas atividades (2 artigos). Não foram encontrados artigos que relacionassem todos os descritores deste estudo, constatando-se, portanto, que havia poucos estudos sobre a temática, principalmente sobre as lacerações.

Isto posto questionou-se: Quais problemas perineais a perineorrafia acarreta no puerpério imediato? Quais atividades habituais e necessidades fisiológicas são afetadas pela presença da perineorrafia no puerpério imediato? Há associação entre a presença da perineorrafia e os problema perineais, as atividades habituais e as necessidades fisiológicas?

Tendo como objeto de investigação: A associação entre perineorrafia/ traumas perineais (episiotomia/episiorrafia ou laceração espontânea) com problemas perineais, atividades habituais, necessidades fisiológicas em puérperas com parto vaginal.

Assim, este estudo teve como objetivo geral verificar a associação entre a presença de perineorrafia e problemas perineais, atividades habituais e necessidades fisiológicas afetadas em puérperas no pós-parto vaginal em uma maternidade pública de Feira de Santana, Bahia, Brasil.

\section{MATERIAIS E MÉTODOS}

Trata-se de um estudo do tipo transversal, realizado em uma maternidade pública do município de Feira de Santana, Bahia, Brasil, 
na unidade de alojamento conjunto, durante os meses de setembro de 2012 a dezembro de 2014. O referido local do estudo consiste numa instituição hospitalar que fornece assistência a mulheres durante o trabalho de parto, parto e puerpério e a recém nascidos saudáveis e em processo de adoecimento.

A população deste estudo foi composta por 1524 mulheres, sendo que 870 (57\%) compuseram a amostra. Para a seleção das puérperas que compuseram a amostra, foram utilizados os seguintes critérios de inclusão: ser puérpera de parto normal e em vértice que tenha apresentado alguma lesão perineal decorrente do processo parturitivo; puérpera com condições psíquicas, clínicas e obstétricas adequadas para responder aos questionamentos dos pesquisadores; puérperas com mais de seis horas de pós-parto.

Foram excluídas as puérperas que apresentaram episiotomias e lacerações perineais espontâneas concomitantemente, bem como aquelas com lacerações não suturadas, pela possibilidade destas lesões não acarretarem problemas perineais devido à ausência de sutura ou não dificultarem a realização de alguma atividade habitual ou necessidade fisiológica da puérpera.

Os dados foram coletados nos turnos matutino e vespertino, de segunda a sexta-feira, durante o período anteriormente citado. Foi utilizada a técnica de entrevista estruturada, durante a qual também foi utilizado um formulário para coleta de informações para identificação das entrevistadas. As informações coletadas para a identificação foram relacionadas às condições gestacionais, obstétricas e perinatais; tipo de trauma perineal, principais problemas decorrentes da presença destes traumas, com destaque para a dor, e as atividades habituais e necessidades fisiológicas que foram dificultadas. Além disso, foi realizado o exame físico da região vulvoperineal e a mensuração da intensidade da dor através da Escala Numérica Compartimentada, composta por uma pontuação que varia de 0 à 10 pontos.

Como variáveis de exposição, foram utilizadas a episiorrafia (sutura de episiotomia) e a perineorrafia secundária à laceração espontânea, consideradas, de uma forma geral, como perineorrafia, pois consistem em suturas de lesões perineais. As variáveis de desfecho, por sua vez, foram os problemas perineais (edema, eritema, ardor, dor e hematoma), as atividades habituais (deambular, amamentar, sentar e vestir-se) e as necessidades fisiológicas (dormir, alimentar-se, higiene íntima, urinar e evacuar).

Os dados foram analisados por meio do pacote StatisticalPackagefor the Social Sciences(SPSS), versão 22.0, sendo utilizadas as estatísticas descritiva e inferencial. Para a análise descritiva, foi realizada a contagem das frequências simples. Para a análise inferencial, por sua vez, a fim de se estabelecer a associação entre as variáveis de exposição e desfecho, foi empregado o teste Qui-quadrado de Pearson e nível de significância de $5 \%(p<0,05)$, calculando-se as razões de prevalência (RP) e seus respectivos intervalos de confiança de $95 \%$.

Esta pesquisa respeitou os aspectos éticos da Resolução no 466/12 do Conselho Nacional de Saúde que envolve pesquisas com seres humanos, e foi aprovada sob o parecer de número 842.198. Sendo assim, as participantes do estudo foram convidadas a participar e informadas, antes da coleta, acerca dos objetivos, riscos e benefícios aos quais as mesmas estariam sujeitas, bem como 
sobre a preservação do seu anonimato, através da leitura do Termo de Consentimento Livre e Esclarecido, que foi devidamente assinado em duas vias, ficando uma com o entrevistado e outra com os pesquisadores.

\section{RESULTADOS}

De acordo com a tabela $1,87,6 \%$ das mulheres com episiotomia tinham idade até 30 anos, 37,9\% estudaram até o ensino médio completo, 37,2\% se autodeclararam pardas, $78 \%$ eram primíparas e $67,1 \%$ realizaram 6 ou mais consultas de prénatal.

Entre mulheres com perineorrafia secundária a laceração espontânea, por sua vez, 45,5\% tinha idade entre 21 e 30 anos, 53,2\% possuíam ensino médio completo, $45,4 \%$ e $33,8 \%$ eram, respectivamente, primíparas e secundíparas, e $61,2 \%$ além de terem realizado seis ou mais consultas de pré-natal Tabela 1.

Tabela 1. Frequências das variáveis sociodemográficos e gestacionais das mulheres que tiveram parto vaginal e sofreram traumas perineais em uma instituição pública, Feira de Santana (BA) set/2012-dez/2014

\begin{tabular}{|c|c|c|c|c|}
\hline Variáveis & $\begin{array}{c}\text { Episiotomia } \\
\text { N (441) }\end{array}$ & $\%$ & $\begin{array}{c}\text { Laceração } \\
\text { N (429) }\end{array}$ & $\%$ \\
\hline \multicolumn{5}{|l|}{ Idade } \\
\hline Abaixo de 20 anos & 208 & 47,2 & 135 & 31,5 \\
\hline De 21 a 30 anos & 178 & 40,4 & 195 & 45,5 \\
\hline Maior igual a 31 anos & 55 & 12,5 & 99 & 23,1 \\
\hline \multicolumn{5}{|l|}{ Grau de escolaridade } \\
\hline Não sabe ler ou escrever & 5 & 1,1 & 8 & 1,9 \\
\hline Fundamental incompleto & 131 & 29,7 & 118 & 27,5 \\
\hline Fundamental completo & 25 & 5,7 & 33 & 7,7 \\
\hline Ensino médio incompleto & 106 & 24 & 86 & 20 \\
\hline Ensino médio completo & 154 & 34,9 & 173 & 40,3 \\
\hline Superior incompleto & 12 & 2,7 & 10 & 2,3 \\
\hline Superior completo & 8 & 1,8 & 1 & 0,2 \\
\hline \multicolumn{5}{|l|}{ Raça/cor } \\
\hline Pretas & 167 & 37,9 & 153 & 35,7 \\
\hline Pardas & 226 & 51,2 & 231 & 53,2 \\
\hline Brancas & 36 & 8,2 & 32 & 7,5 \\
\hline Amarela & 10 & 2,3 & 11 & 2,6 \\
\hline Indígenas & 2 & 0,5 & 2 & 0,5 \\
\hline \multicolumn{5}{|l|}{ Número de partos } \\
\hline 1 parto & 344 & 78 & 196 & 45,7 \\
\hline 2 partos & 73 & 16,6 & 145 & 33,8 \\
\hline Três ou mais partos & 24 & 5,4 & 88 & 20,5 \\
\hline \multicolumn{5}{|c|}{ Número de consulta pré-natal } \\
\hline Máximo de 5 consultas & 145 & 32,9 & 166 & 38,8 \\
\hline 6 ou mais consultas & 296 & 67,1 & 262 & 61,2 \\
\hline
\end{tabular}

Fonte: Banco de dados do Projeto de Pesquisa intitulado "Condições perineais de mulheres no pósparto vaginal em uma Instituição pública do interior da Bahia”, 2015. 
Conforme evidenciado na Tabela $2,57,1 \%$ das mulheres que tiveram episiotomia permaneceram no centro obstétrico por até 5 horas da admissão na maternidade (média de 6,1 horas, $\pm 5,1$ ), em $61,7 \%$ delas foi administrada ocitocina intravenosa durante o trabalho de parto, 97,5\% utilizaram a postura horizontal durante o parto e $43,2 \%$ destes partos ocorreram no período noturno. Quanto ao tipo de episiotomia, 66,3\% foi médio lateral esquerda Tabela 2.

No grupo das mulheres que apresentaram laceração, 64,6\% ficaram até 5 horas no centro obstétrico (média de 5,7 horas, $\pm 7,2$ ), 58,7\% receberam ocitocina intravenosa. Quanto ao grau da laceração, 53,3\% foram classificadas como de segundo grau Tabela 2.

Tabela 2. Frequências das variáveis obstétricas das mulheres que tiveram parto vaginal com episiotomia e laceração, em uma instituição pública, Feira de Santana (BA), set/2012-dez/2014

\begin{tabular}{|c|c|c|c|c|}
\hline Variáveis & $\begin{array}{c}\text { Episiotomia } \\
\text { N (441) }\end{array}$ & $\%$ & $\begin{array}{l}\text { Laceração } \\
\text { N (429) }\end{array}$ & $\%$ \\
\hline \multicolumn{5}{|l|}{ Tempo de internamento } \\
\hline Até 5 horas & 252 & 57,1 & 277 & 64,6 \\
\hline 6 á 10 horas & 114 & 25,9 & 101 & 23,5 \\
\hline Acima de 11 horas & 75 & 17 & 51 & 11,9 \\
\hline \multicolumn{5}{|c|}{ Uso de ocitocina intravenosa } \\
\hline Sim & 272 & 61,7 & 252 & 58,7 \\
\hline Não & 169 & 38,3 & 176 & 41,3 \\
\hline \multicolumn{5}{|l|}{ Tipo de episiotomia* } \\
\hline Mediana & 20 & 4,6 & - & - \\
\hline Médio lateral direita & 128 & 29,2 & - & - \\
\hline Médio lateral esquerda & 291 & 66,3 & - & - \\
\hline \multicolumn{5}{|l|}{ Grau da laceração } \\
\hline $1^{\circ} \mathrm{Grau}$ & - & - & 127 & 32,1 \\
\hline $2^{\circ} \mathrm{Grau}$ & - & - & 219 & 55,3 \\
\hline $3^{\circ} \mathrm{Grau}$ & - & - & 46 & 11,6 \\
\hline $4^{\circ} \mathrm{Grau}$ & - & - & 4 & 1 \\
\hline
\end{tabular}

*13 mulheres recusaram o exame físico no períneo devido à intensa dor local e não havia sido anotado no prontuário o tipo de episiotomia realizada.

Fonte: Banco de dados do Projeto de Pesquisa intitulado "Condições perineais de mulheres no pósparto vaginal em uma Instituição pública do interior da Bahia”, 2015.

No que concerne aos problemas perineais, observou-se diferenças estatisticamente significativas entre os grupos com episiorrafia e perineorrafia secundária a laceração quanto a ocorrência de edema, ardor e dor. Tabela 3.
A prevalência de edema perineal foi de 1,7 vezes maior $(p<0,001)$ entre as mulheres com epi siorrafia, assim como a de relato de dor local foi de 1,1 vezes maior $(p=0,002)$. Entretanto, houve uma prevalência menor de relatos de 
ardor $(\mathrm{RP}=0,007 ; \mathrm{p}=0,002)$ entre as puérperas deste mesmo grupo. A prevalência de eritema perineal nas bordas da sutura, por sua vez, foi de 3,1 vezes maior entre as mulheres com episiorrafia ( $p=0,34)$, mas essa relação não foi estatisticamente significativa. Tabela 3.

Tabela 3. Associação entre perineorrafia com problemas perineais em puérperas com parto vaginal em uma maternidade pública, Feira de Santana (BA), set/2012-dez/2014

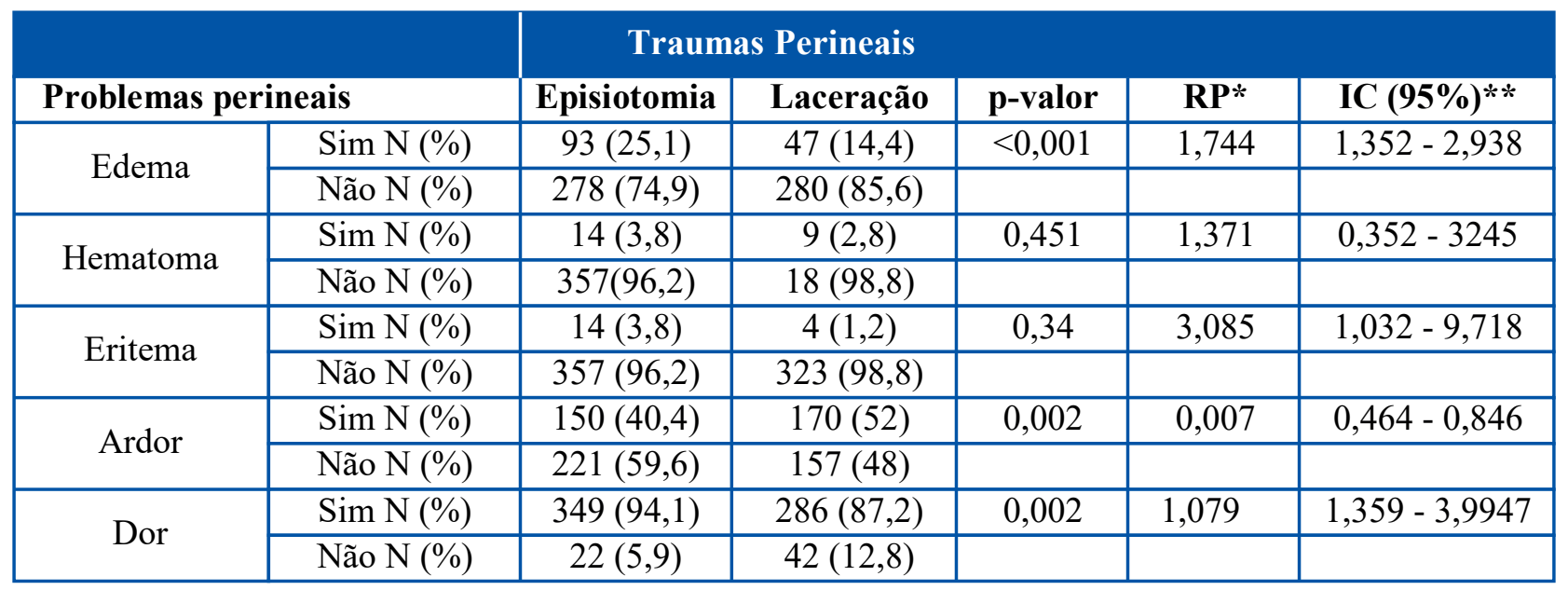

RP: Razão de Prevalência.

** IC: Intervalo de Confiança.

Fonte: Banco de dados do Projeto de Pesquisa Intitulado "Condições perineais de mulheres no pósparto vaginal em uma Instituição pública do interior da Bahia”, 2015.

Concernente às atividades habituais, mulheres com episiorrafia apresentaram maior dificuldade para deambular $(\mathrm{RP}=1,2 ; \mathrm{p}=0,002)$, quando comparadas àquelas com sutura de lacerações perineais. Em contrapartida, com relação às necessidades fisiológicas, apresentaram maior dificuldade para dormir $(\mathrm{RP}=1,2 ; \mathrm{p}<0,001)$ e menor dificuldade para urinar $(\mathrm{RP}=0,8 ; \mathrm{p}=0,002$; IC: 0,457-0,834) e realizar higiene íntima $(\mathrm{RP}=0,725 ; \mathrm{p}<0,001 ; \mathrm{IC}: 0,429-0,789)$, sendo que, portanto, as mulheres com perineorrafia secundária a laceração apresentaram maior prevalência destas duas últimas dificuldades Tabela 4.

Não foi observada diferença estatística entre os grupos quanto à atividade de amamentar e às necessidades fisiológicas de alimentar-se e evacuar. Contudo, as puérperas com episiorrafia, apesar de apresentarem uma baixa frequência de relatos de dificuldade para evacuar $(9,5 \%)$, em comparação com as mulheres com perineorrafia secundária à laceração, apresentaram maior dificuldade para o atendimento desta necessidade Tabela 4. 
Tabela 4. Associação entre perineorrafia com as atividades habituais e necessidades fisiológicas em puérperas com parto vaginal em uma maternidade pública, Feira de Santana (BA), set/2012$\operatorname{dez} / 2014$

\begin{tabular}{|c|c|c|c|c|c|c|}
\hline & \multicolumn{2}{|c|}{ Traumas Perineais } & & & \\
\hline & & \multirow[t]{2}{*}{ Episiotomia } & \multirow[t]{2}{*}{ Laceração } & & & \\
\hline \multicolumn{2}{|c|}{ Atividades habituais } & & & p-valor & $\mathbf{R P} *$ & IC $(95 \%) * *$ \\
\hline \multirow[t]{2}{*}{ Amamentar } & Sim N (\%) & $55(14,6)$ & $287(89,1)$ & 0,143 & 1,342 & $0,891-2,202$ \\
\hline & Não N (\%) & $322(85,4)$ & $35(10,9)$ & & & \\
\hline \multirow[t]{2}{*}{ Deambular } & Sim N (\%) & $232(61,5)$ & $161(50)$ & 0,002 & 1,231 & $1,184-2,163$ \\
\hline & Não N (\%) & $145(38,5)$ & $161(50)$ & & & \\
\hline \multirow[t]{2}{*}{ Sentar } & Sim N (\%) & $324(85,8)$ & $258(80,1)$ & 0,040 & 1,073 & $1,017-2,260$ \\
\hline & Não N (\%) & $53(14,1)$ & $64(19,9)$ & & & \\
\hline \multirow[t]{2}{*}{ Vestir - se } & Sim N (\%) & $85(22,5)$ & $53(16,5)$ & 0,044 & 1,370 & $1,009-2,162$ \\
\hline & Não N (\%) & $292(77,5)$ & $269(83,5)$ & & & \\
\hline \multicolumn{7}{|c|}{ Necessidades fisiológicas } \\
\hline \multirow[t]{2}{*}{ Dormir } & Sim N (\%) & $127(33,7)$ & $70(21,7)$ & $<0,001$ & 1,150 & $1,301-2,570$ \\
\hline & Não N (\%) & $250(66,3)$ & $252(78,3)$ & & & \\
\hline \multirow[t]{2}{*}{ Alimentar-se } & Sim N $(\%)$ & $14(3,7)$ & $8(2,5)$ & 0,354 & 1,495 & $0,627-3,656$ \\
\hline & Não N (\%) & $363(96,3)$ & $314(97,5)$ & & & \\
\hline \multirow[t]{2}{*}{ Urinar } & Sim N (\%) & $145(38,5)$ & $162(50,3)$ & 0,002 & 0,764 & $0,457-0,834$ \\
\hline & Não N (\%) & $232(61,5)$ & $160(49,7)$ & & & \\
\hline \multirow[t]{2}{*}{ Higiene íntima } & Sim N (\%) & $129(34,2)$ & $152(47,2)$ & $<0,001$ & 0,725 & $0,429-0,789$ \\
\hline & Não N (\%) & $248(65,8)$ & $170(40,7)$ & & & \\
\hline \multirow[t]{2}{*}{ Evacuar } & Sim N (\%) & $36(9,5)$ & $18(5,6)$ & 0,051 & 1,708 & $0,992-3,205$ \\
\hline & Não N (\%) & $341(90,5)$ & $304(94,4)$ & & & \\
\hline
\end{tabular}

RP: Razão de Prevalência.

** IC: Intervalo de Confiança.

Fonte: Banco de dados do Projeto de Pesquisa intitulada "Condições perineais de mulheres no pósparto vaginal em uma Instituição pública do interior da Bahia”, 2015.

\section{DISCUSSÃO}

A maioria das mulheres com episiorrafia e perineorrafia secundária à laceração espontânea apresentaram algum problema perineal ou relataram dificuldades para realizar algumas de suas atividades habituais ou atender as suas necessidades fisiológicas.

Dentre os problemas perineais, observou-se diferença estatisticamente significativa quanto a ocorrência de edema, ardor e dor entre os grupos, sendo que as mulheres com episiorrafia relataram menos ardor perineal em comparação ao grupo de puérperas com perineorrafia devido a laceração espontânea. Isso pode ser justificado pelo local de ocorrência do tipo da laceração, pois pode atingir locais de maior exposição e sensibilidade para esse sintoma.

No que concerne à dor, tanto a literatura nacional quanto a internacional apontam elevada frequência deste sintoma associado à perineorrafia, que varia de $18,5 \%$ a $90 \%{ }^{13-21}$. 
Um realizado com 143 mulheres, encontrou que puérperas com episiorrafia relataram duas vezes mais dor perineal do que as que apresentaram períneo íntegro após o parto vaginal, destacando o incômodo gerado pelas altas taxas de episiotomia $^{17}$. Nesse sentido, preservar a integridade perineal das parturientes é algo necessário e possível no cenário do cuidado obstétrico, visto que esse tipo de intervenção, por vezes desnecessária, desencadeará desconfortos que irão interferir no seu bem-estar durante o período puerperal.

Com relação à classificação da dor, observou-se, neste estudo, maior prevalência da intensidade moderada, sendo este achado semelhante ao de outros estudos ${ }^{15-16}$.

Entretanto, observa-se que os estudos anteriormente mencionados apresentaram prevalência da dor perineal comparando mulheres com episiorrafia e períneo íntegro, o que difere do atual estudo.

Um outro estudo traz que mulheres com dor em decorrência de traumas perineais apresentam quatro vezes mais dor, em comparação àquelas com períneo íntegro. Contudo nos grupos com traumas perineais, não houve diferença significante quanto à intensidade de dor, além de que primíparas com menor tempo de pós-parto referiram dor perineal mais intensa ${ }^{21}$.

Outro estudo, entretanto, demonstrou que mulheres com dor em decorrência da perineorrafia classificaram-na, em sua maioria, como leve (53\%), confrontando com os achados do presente estudo $^{19}$.
No que concerne ao edema, o atual estudo verificou que a episiorrafia eleva em 1,7 vezes a prevalência de a mulher apresentar edema. Um estudo com uma amostra de 303 mulheres corrobora este achado, uma vez que 37,5\% das mulheres que pertenceram ao estudo tiveram edema em decorrência deste tipo de perineorrafia $^{15}$.

Esses dados também são semelhantes a outro estudo realizado com 54 puérperas, visto que $44 \%$ destas apresentaram edema como complicação perineal nas primeiras 48 horas do pós-parto ${ }^{11}$.

Outro estudo revelou que a dor em decorrência do edema da tende a limitar as atividades habituais, como sentar e deambular, exercidas pelas mulheres ${ }^{8}$. Outros sinais e sintomas e comorbidades podem estar associadas a episiorrafia, tais como a equimose, o hematoma e a infecção local ${ }^{22}$. Nesta pesquisa, observou-se baixa frequência de hematoma no local da sutura perineal, que apesar de apresentar diferença significativamente estatística entre os grupos, foi mais frequente entre as puérperas com episiorrafia.

Os traumas perineais, de uma forma geral, sendo intencionais ou não, podem causar na mulher algum tipo de exposição, uma vez que rompem com a barreira de continuidade da pele causando alteração tissular. Essas alterações podem ser de ordem fisiológicas como os sintomas de dor, ardor, vermelhidão e edema gerados pelo próprio processo de cicatrização, como também complicações patológicas, que a depender do tipo e local que esses vierem a ocorrer poderão colocar em risco a vida da puérpera devido a ocorrência de infecção e/ou hemorragias. 
As mulheres com episiorrafia, na presente pesquisa, relataram menos ardor perineal e dificuldade para urinar e realizar higiene íntima. Um estudo realizado na cidade de São Paulo com mulheres que sofreram laceração espontânea encontrou que mulheres com este tipo de trauma apresentaram ardor na vulva ao urinar $^{22}$. Isso provavelmente se deve por causa do local no qual este tipo de trauma perineal pode ocorrer no períneo anterior.

Lacerações da região anterior do períneo ocorreram em 48\% das mulheres investigadas, sendo mais frequentes aquelas encontradas na região vestibular ${ }^{10}$. Nesse mesmo estudo apenas quatro mulheres apresentaram este tipo de trauma perineal no clitóris. Os mesmos atores chamam atenção para traumas nestes locais, por ser uma área muito sensível e vascularizada, podendo causar dor tanto durante a sutura como no puerpério imediato.

Pensa-se que a maior tensão utilizada para a realização da episiorrafia poderá ocasionar dor perineal no puerpério imediato, além do hematoma e do edema na região ao redor da sutura. Isso pode estar associado a maior necessidade de reação inflamatória exigida pelo organismo da puérpera nos primeiros dias após o parto, acarretando maior acúmulo de fluídos no local da sutura perineal.

Uma revisão sistemática que objetivou estudar a sutura em laceração de primeiro e segundo graus encontrou que as suturas em lacerações de primeiro grau podem estar mais associadas a problemas perineais e mudanças na realização de atividades durante o puerpério imediato do que aquelas que não foram suturadas ${ }^{22}$.
Esses problemas perineais podem desencadear dificuldadespararealizaçãodeatividadeshabituais e o atendimento de necessidades fisiológicas nos primeiros dias após o parto. Na presente pesquisa associação estatisticamente significante entre episiorrafia e estas alterações. As mulheres com este tipo de perineorrafia relataram maior dificuldade para vestir-se, deambular, sentar, dormir e evacuar quando comparada com as que apresentaram perineorrafia devido às lacerações espontâneas no parto.

Ao realizar um estudo com 50 mulheres sobre dor em decorrência da episiotomia constatouse que, as atividades mais modificadas referidas pelas usuárias foram sentar, deitar, deambular, urinar, evacuação, higiene íntima e dormir ${ }^{8}$.

Esses dados compartilham com os achados estatísticos deste estudo, tendo em vista que a presença da episiotomia aumenta a dificuldade das mulheres para dormir nas primeiras horas do período pós-parto.

Desta forma, fica evidenciado cientificamente o comprometimento das atividades habituais por ocorrência dos traumas perineais, por conseguinte a mulher poderá sofrer com essa condição, pois é no período puerperal que ela precisa estar bem e apta para desenvolver ações de autocuidado e cuidados com o seu (a) recém-nascido (a).

Um estudo sobre a prevalência de dor para realizar atividades habituais e necessidades fisiológicas observou que a dor perineal em decorrência da perineorrafia causa em algumas mulheres dificuldade para amamentar, sentar, deambular e realizar higiene íntima ${ }^{19}$. 
Diante de todas as questões expostas, entende-se que é imprescindível a adoção de intervenções que auxiliem na redução dos problemas perineais apresentados pelas mulheres no período do pós-parto vaginal, considerando que esta é uma função do enfermeiro, pois consistem em práticas de cuidado. A redução desses problemas, inclusive, pode resultar em melhora na execução das atividades habituais e das necessidades fisiológicas.

Sendo assim, algumas dessas intervenções poderiam ser medicamentosas, com a administração de analgésicos e antiinflamatórios previamente prescritos ${ }^{23}$, e não medicamentosas, a exemplo da crioterapia, que auxilia na redução da dor e promove sensação de conforto às puérperas $^{24}$.

Considera-se, que este estudo possui algumas limitações. Na literatura nacional e internacional inexistem estudos sobre a associação entre perineorrafias e ocorrência de problema perineais, atividades habituais e necessidades fisiológicas. Dessa forma, este estudo limitouse às comparações destas condições com estudos sobre a episiorrafia. Além disso uma outra limitação é o fato de este estudo ser do tipo transversal, visto que observa o evento em apenas um dado momento do tempo, sem poder identificar os fatos precedentes à ocorrência.

\section{CONCLUSÕES}

Os resultados deste estudo permitiram verificar que a presença de problemas perineais e as dificuldades na realização de atividades habituais e no atendimento às necessidades fisiológicas ocorrem de forma distinta entre as mulheres com perineorrafia secundária à laceração e à episiotomia.
Foi possível inferir que mulheres com episiorrafia apresentaram maior ocorrência de edema e dor, ao passo que possuíram menor tendência para relato verbal de ardor quando comparadas às mulheres com perineorrafia secundária à laceração. No que se refere às atividades habituais, percebeuse que o mesmo grupo de puérperas relataram maior dificuldade para deambular e dormir, ao passo que, concernente ao atendimento das necessidades fisiológicas, referiram menor dificuldade para urinar e realizar higiene íntima, sendo estas, portanto, mais frequentes entre as puérperas com o outro tipo de perineorrafia. Dessa forma, evidencia-se que puérperas com perineorrafia de diferentes etiologias podem apresentar diferentes demandas relacionadas às variáveis de desfecho pesquisadas.

Ademais, tendo em vista todas as implicações associadas à episiorrafia, os resultados demostram a importância da restrição do uso da episiotomia, além de ser imperativa a implementação de técnicas de proteção ao períneo durante o trabalho de parto como forma de prevenir a laceração perineal.

O estudo científico, como o que foi proposto neste estudo é algo seletivo onde o seu foco é direcionado para a observação do que é relevante, detectando o que há de mais significativo durante a análise ${ }^{25}$. Com isso sugere-se a realização de novas investigações que contemplem algumas das limitações deste estudo e que as mesmas possam ser realizadas em outras regiões do país, para fins de comparações.

Conflitos de interesse: Os autores declaram que não houve conflitos de interesses. 


\section{REFERENCIAS}

1. Mattar R, Aquino MMA, Mesquita MRS. A prática da episiotomia no Brasil. Rev Bras Ginecol Obstet. 2007; 29(1): $1-2$. https://doi.org/10.1590/S0100-72032007000100001

2. Santos LM, Pereira SSC. Vivências de mulheres sobre a assistência recebida no processo parturitivo. Physis. 2012; 22(1): 77-97.

http://dx.doi.org/10.1590/S0103-73312012000100005

3. Leal MC. Pesquisa nascer no brasil: inquérito sobre parto e nascimento. Disponível em:

http://www.conass.org.br/sintese_pesquisa_nascer_brasil. pdf

4. Muhleman MA, Aly I, Walters A, Topale N, Tubbs RS, Loukas M. Cut or Not to Cut, That is the Question: A Review of the Anatomy, the Technique, Risks, and Benefits of an Episiotomy. Clinical Anatomy. 2017; 30(3): 362-72. https://doi.org/10.1002/ca.22836

5. Singh S, Thakur T, Chandhiok N, Dhillon BS. Padrão de uso de episiotomia e suas complicações imediatas entre partos vaginais em 18 hospitais terciários na Índia. Indian J Med Res. 2016; 143(4): 474-80. https://doi.org/10.4103/0971-5916.184304

6. Moore KL, Dalley AF, Agur AMR. Anatomia orientada para a clínica. 7. ed. Rio de Janeiro (RJ): Guanabara Koogan; 2014.

7. Cichowski S, Rogers R. Boletim de Prática $N^{\circ} 165$ : Prevenção e Gerenciamento de Lacerações Obstétricas no Parto Vaginal. Comitê de Boletins de Prática do Colégio Americano de Obstetras e Ginecologistas - Obstetrícia. Obstet Gynecol. 2016; 128(1): 1-15. http://doi.org/10.1097/ AOG.0000000000001523

8. Beleza ACS, Ferreira CHJ, Sousa L, Nakano AMS. Mensuração e caracterização da dor após episiotomia e sua relação com a limitação de atividades. Rev Bras Enferm. 2012; 65(2): 264-8.

http://dx.doi.org/10.1590/S0034-71672012000200010

9. Macarthur AJ, Macarthur C. Incidence, severity, and determinants of perineal pain after vaginal delivery: a prospective cohort study. Am J Obstet Gynecol. 2004; 191(40): 1199-204.

https://doi.org/10.1016/j.ajog.2004.02.064

10. Caroci AS, Riesco MLG, Leite JS, Araújo NM, Scarabotto LB, Oliveira SMJV. Localização das lacerações perineais no parto em mulheres primíparas. Rev Enferm UERJ. 2014; 22(3): 402-8. http://dx.doi.org/10.1590/S1519-38292009000400004

11. Alvarenga MB, Francisco AA, Vasconcelos SMJ, Barbosa FM, Shimoda GT, Damiani LP, et al. Avaliação da cicatrização da episiotomia: confiabilidade da escala REEDA (Redness, Oedema, Ecchymosis, Discharge, Approximation). Rev Latino-Am Enfermagem. 2015; 23(1): 162-8.

https://doi.org/10.1590/0104-1169.3633.2538

12. Pereira da Silva JC, Soler ZASG, Wysocki AD. Fatores associados à incontinência urinária em mulheres submetidas ao exame urodinâmico. Rev Esc Enferm USP. 2017; 51(3): 1-09.

http://dx.doi.org/10.1590/s1980-220x2016140903209
13. Cesar MAP, Leite JM, Muniz RCC, Ortiz JA. Distúrbios evacuatórios em primigestas após parto normal: estudo clínico. Rev Bras Coloproct 2011; 31(2): 126-30. http://dx.doi.org/10.1590/S0101-98802011000200003

14. Leeman L, Fullilove AM, Borders N, Manocchio R, Albers LL, Rogers RG. Postpartum perineal pain in a low episiotomy setting: association with severity of genital trauma, labor care, and birth variables. Birth. 2009; 36(4): 283-8. https://10.0.4.87/j.1523-536X.2009.00355.x

15. Amorim FA, Junqueira VOSM, Barbosa SFM, Bick D, Gonzalez RML. Women's experiences of perineal pain during the immediate postnatal period: cross-sectional study in Brazil. Midwifery. 2011; 27(6): 254-9.

https://doi.org/10.1016/j.midw.2010.10.012

16. Francisco AA, Oliveira SMJV, Santos JO, Silva FMB. Avaliação e tratamento da dor perineal no pós-parto vaginal. Acta Paul Enferm. 2011; 24(1): 94-100.

http://dx.doi.org/10.1590/S0103-21002011000100014

17. Imarengiaye CO, Andet AB. Postpartum perineal pain among Nigerian women. West African journal of medicine. 2008; 27(3): 148-51.

18. Almeida CB, Sé CCS, Pereira EG, Pereira ALF. Avaliação da dor decorrente da perineorrafia no parto normal. $R$. pesq.: cuid. fundam. 2011; 3(3): 2126-36.

19. East CE, Sherburn M, Nagle C, Said J, Forster D. Perineal pain following childbirth: Prevalence, effects on postnatal recovery and analgesia usage. Midwifery. 2012; 28(1): 93-7. http://10.1016/j.midw.2010.11.009

20. Santos JO, Oliveira SM, Nobre MR, Aranha AC, Alvarenga MB. A randomised clinical trial of the effect of low-level laser therapy for perineal pain and healing after episiotomy: A pilot study. Midwifery. 2012; 28(5): 653-9. http://10.1016/j.midw.2011.07.009

21. Francisco AA, Kinjo MH, Bosco CS, Silva RL, Mendes EPB, Oliveira SMJV. Associação entre trauma perineal e dor em primíparas. Revista da Escola de Enfermagem da USP. 2014; 48(1): 40-5.

http://dx.doi.org/10.1590/S0080-623420140000600006

22. Elharmeel SM, Chaudhary Y, Tan S, Scheermeyer E, Hanafy A, Van ML. Surgical repair of spontaneous perineal tears that occur during childbirth versus no intervention. Cochrane Database Syst. Rev. Library. 2015; 14(8).

23. Peleckis MV, Francisco AA, Oliveira SMJV. Terapias de alívio da dor perineal no pós-parto. Texto Contexto Enferm. 2017; 26(2).

http://dx.doi.org/10.1590/0104-07072017005880015

24. Francisco AA, Oliveira SMJV, Steen M, Nobre MRC, Souza EV. Ice pack induced perineal analgesia after spontaneous vaginal birth: Randomized controlled trial. Women Birth. 2018.

https://doi.org/10.1016/j.wombi.2017.12.011

25. Cañón-Montañez W. El método científico en las ciencias de la salud. Rev Cuid. 2011; 2(1): 94-5.

https://doi.org/10.15649/cuidarte.v2i1.43 